Article type : Original Article

\title{
The projected burden of osteoarthritis and rheumatoid arthritis in Australia: A population-level analysis
}

Ilana N Ackerman: BPhysio(Hons), PhD

Monash University, Melbourne, Australia; and The University of Melbourne, Melbourne, Australia

Clare Pratt: BSc, BA

Formerly Department of Medicine (Royal Melbourne Hospital), The University of Melbourne, Australia

Alexandra Gorelik: BA, MSc

Melbourne EpiCentre, Royal Melbourne Hospital and The University of Melbourne, Australia

Danny Liew: MBBS(Hons), BMedSc, FRACP, PhD, CertHealthEcon

Monash University, Melbourne, Australia; and The University of Melbourne, Melbourne, Australia

\section{Correspondence to:}

Associate Professor Ilana Ackerman

Department of Epidemiology and Preventive Medicine, Monash University

The Alfred Centre, 99 Commercial Road, Melbourne, Victoria 3004, Australia

Phone: +61399030585 Fax: +61399030556

Email: ilana.ackerman@monash.edu

\section{Running head}

Projected burden of arthritis in Australia

\section{Word count}

This is the author manuscript accepted for publication and has undergone full peer review but has not been through the copyediting, typesetting, pagination and proofreading process, which may lead to differences between this version and the Version of Record. Please cite this article as doi: $\underline{10.1002 / a c r .23414}$

This article is protected by copyright. All rights reserved 


\section{Funding}

This work was supported by funding from Arthritis Australia, made possible by an unrestricted grant from the Medicines Australia Community Chest to Arthritis Australia. The Community Chest supporters are AbbVie Pty Ltd, AstraZeneca Pty Ltd, Eli Lilly Australia Pty Ltd, Janssen-Cilag Pty Ltd, Pfizer Australia Pty Ltd, and Roche Products Pty Ltd. Associate Professor Ilana Ackerman was supported by a National Health and Medical Research Council of Australia Public Health (Australian) Early Career Fellowship (\#520004).

\section{Abstract}

Objective: To forecast the prevalence and direct healthcare costs of osteoarthritis (OA) and rheumatoid arthritis (RA) in Australia to the year 2030.

Methods: An epidemiological model of the Australian population was developed. Data on the national prevalence of $O A$ and RA were obtained from the Australian Bureau of Statistics (ABS) 2014-2015 National Health Survey. Future prevalence was estimated using ABS population projections for 2020, 2025 and 2030. Available government data on direct healthcare expenditure for OA and RA were modelled to forecast costs (in AUD) for the years 2020, 2025 and 2030, from the perspective of the Australian public healthcare system.

Results: The number of people with $O A$ is expected to increase nationally from almost 2.2 million in 2015 to almost 3.1 million Australians in 2030. The number of people with RA is projected to increase from 422,309 in 2015 to 579,915 in 2030 . Healthcare costs for OA were estimated to be over $\$ 2.1$ billion in 2015 ; by the year 2030 , these are forecast to exceed $\$ 2.9$ billion ( $\$ 970$ for every person with the condition). Healthcare costs for RA were estimated to be over $\$ 550$ million in 2015 , including $\$ 273$ million spent on biological disease-modifying anti-rheumatic drugs. Healthcare costs for RA are projected to rise to over $\$ 755$ million by the year 2030 .

Conclusions: $O A$ and RA are costly conditions that will impose an increasing healthcare burden at the population level. These projections provide tangible data that can be utilised to map future health service provision to expected need.

\section{Word count: 249}

This article is protected by copyright. All rights reserved 
Keywords: Epidemiology; Health Care Costs; Osteoarthritis; Prevalence; Rheumatoid arthritis

\section{Significance and Innovations}

- Understanding the prevalence and costs of chronic conditions such as osteoarthritis (OA) and rheumatoid arthritis (RA) is vital for governments and other healthcare funders, to ensure that healthcare services are capable of meeting current and future population demand

- Projections of growth can be used to plan appropriate resource allocation and to plan future health workforce requirements

- By 2030, nearly 3.1 million Australians are predicted to have OA and from a health system perspective, the direct healthcare costs for OA are forecast to exceed \$AUD 2.9 billion

- Direct healthcare costs for RA are anticipated to exceed \$AUD 755 million by 2030, and this relates substantially to the costs associated with biological therapies

\section{Introduction}

Osteoarthritis $(\mathrm{OA})$ is the most common form of arthritis in Australia (1) and according to the landmark Global Burden of Disease study, a major cause of years lived with disability (YLD) globally (2). Specifically, knee and hip OA exert a significant impact on health systems internationally, given the substantial costs related to joint replacement surgery for these conditions. In 2010, the global age-standardised prevalence of knee and hip OA was $3.8 \%$ and $0.85 \%$, respectively, and over 17 million YLDs were attributable to hip and knee OA collectively (3). RA is the second most common form of arthritis in Australia (1). Globally, the prevalence of RA was estimated to be $0.24 \%$ and the condition was associated with almost 3.8 million YLDs in 2010 (4).

A range of national and international guidelines have been developed to support the timely and effective management of $\mathrm{OA}$ and $\mathrm{RA}$ (5-12). OA guidelines routinely advocate non-surgical approaches (including weight loss, exercise, disease-relevant education and self-management support) as core treatment strategies, with referral for consideration of joint replacement or other surgery where non-surgical management is no longer effective. In contrast to OA recommendations, pharmacological management is the mainstay of RA treatment. Analgesics and anti-inflammatory are commonly used to treat RA symptoms while disease-modifying antirheumatic drugs (DMARDs) are used to reduce disease activity, limit joint destruction, and induce remission. 
An understanding of arthritis prevalence and associated healthcare costs is vital for governments and other funders of healthcare to ensure that health services are capable of meeting population demand. Projections of growth in arthritis populations over time are also important for planning future health service provision, including appropriate resource allocation and health workforce requirements. To inform macro-level policy decisions, burden of disease analyses should incorporate the most up-to-date national data. While data from the 2004-2005 and 2011-2012 Australian National Health Surveys have informed earlier arthritis prevalence projections $(13,14)$, the most recent iteration (the 2014-2015 National Health Survey) represents a key opportunity to generate contemporary burden of disease estimates. Additionally, previous investigations of arthritis costs in Australia have relied on national health expenditure data from 2000-2001 and have not provided future cost projections based on growing arthritis populations $(13,14)$. Over the past 15 years, rates of joint replacement surgery (performed predominantly for $O A$ ) have continued to rise in Australia $(15,16)$ and treatment strategies for inflammatory arthritis have changed dramatically, largely due to the introduction of new (and expensive) biological therapies (17). These factors undoubtedly have implications for the costs of providing healthcare for arthritis at a population level. Using the most recent data available, our study aimed to forecast the burden of OA and RA in Australia to the year 2030, in terms of disease prevalence and direct healthcare costs.

\section{Methods}

\section{Data sources}

National OA and RA prevalence data were sourced from the Australian Bureau of Statistics (ABS) 2014-2015 National Health Survey (1). This survey comprised 19,259 participants from 14,723 randomly selected private dwellings across Australia. One adult (aged 18 years and over) and one child (aged 0-17 years) from each dwelling were randomly selected for inclusion in the survey. As part of the arthritis module of the survey, participants were asked whether they have or had ever had: gout, rheumatism, arthritis, OA, RA, or other types of arthritis (18). If participants identified an arthritis condition, other than gout or rheumatism, they were asked whether they had ever been told by a doctor or nurse that they had the condition (18). Information on arthritis conditions was also collected as part of the long-term conditions module of the survey.

Data on the current and projected population of Australia were obtained from ABS National Demographic Statistics (19). This dataset provides population projections for Australia (by age and sex) from 2012 to 2101 . Future population predictions are based on assumptions regarding expected rates of fertility, mortality and migration(20). The ABS considers three potential scenarios in their population projections, pertaining to low, medium, and high population growth. We utilised the medium growth scenario (incorporating medium fertility, medium life expectancy and medium net overseas migration assumptions) for our main analyses. Low and high population growth scenarios were used for a sensitivity analysis (Supplementary material).

This article is protected by copyright. All rights reserved 
Direct healthcare costs were estimated from the perspective of the Australian public healthcare system (universal healthcare is provided in the public healthcare system via the governmentfunded Medicare program). We utilised a national healthcare expenditure report from the Australian Institute of Health and Welfare (AIHW) (21) that provides the most recently-published government data on the healthcare costs of arthritis. The report captures in-hospital costs (for example, medical, surgical and in-hospital medication costs), out-of-hospital medical costs, and prescription medication costs (representing approximately $70 \%$ of total direct healthcare expenditure on arthritis). A limitation of this data source is that it underestimates the cost of prescription medications for inflammatory arthritis, such as DMARDs, which are predominantly prescribed by rheumatologists. This is because pharmaceutical costs data in the AIHW report were calculated using prescribing data from the Bettering the Evaluation and Care of Health $(\mathrm{BEACH})$ survey that were then applied to Pharmaceutical Benefits Scheme (PBS) payments. The BEACH survey collects information from general practitioners across Australia (22). It is a comprehensive snapshot of the conditions addressed and treatment plans prescribed in general practice. However, it does not include medications prescribed by medical specialists. Therefore the full costs of DMARDs for RA may not be captured. Additionally, expenditure on 'Highly Specialised Drugs' used in hospitals (including biological therapies) was not included in the AlHW expenditure report. Biological DMARDs (bDMARDs), in particular, are expensive treatments and excluding them from our analysis would lead to a significant underestimation of RA healthcare costs. To address this, we examined the cost of biologics separately, using government Pharmaceutical Benefits Schedule (PBS) expenditure data for the 2014-2015 financial year (23). PBS expenditure data were obtained for each bDMARD indicated for RA, as summarised in the supplementary material (Table S3).

\section{Ethics approval}

Ethics approvals were not required as all data sources were publically available.

\section{Data analysis}

All analyses were performed using Stata version 13.1 (StataCorp LP, College station, TX, USA) and Microsoft Excel ${ }^{\mathrm{TM}} 2010$ (Microsoft, USA) and all costs are reported in Australian dollars (1 AUD is equivalent to 0.76 USD). The prevalence of $O A$ and RA for each age group was obtained from the National Health Survey. These proportions were then applied to the relevant age group population estimates from the ABS National Demographics Statistics for the years 2015, 2020, 2025 and 2030. Prevalence data for OA and RA are presented as the total number of people with the condition in each year by age group and in total. All prevalence estimates were rounded up to the nearest whole number. 
Government expenditure data for in-hospital medical expenses, out-of-hospital medical expenses and prescription medications were extracted from published 2008-2009 national data (21) and updated to 2015-2016 dollars using weights provided for the Total Health Price Index (THPI) (24). These weights were calculated by the AIHW (based on annual ratios of estimated total national health expenditure at current prices) to estimate total national health expenditure at constant prices. For RA, we replaced the reported costs of prescription medications with new estimates for the costs of bDMARDs plus other medications. To project the cost of bDMARDs for the years 2020, 2025 and 2030, we assumed that these costs would grow in proportion to RA prevalence for each of those years (i.e. a $10 \%$ increase in prevalence would result in a $10 \%$ increase in costs). The costs of prescription medications for RA were calculated based on the total cost of bDMARDs plus other medications (for example, conventional DMARDS, analgesics and anti-inflammatory medications). The cost of other medications was derived from published national pharmaceutical expenditure data for RA (21) and assumed to be \$171,782,965 in 2015 (\$132 million in 2008-2009 dollars, updated to 2015-2016 dollars). Applying the projected prevalence estimates, the national costs of healthcare for $O A$ and RA were calculated for each age group according to the following formula (all cost estimates were rounded up to the nearest whole number):

Cost of disease per age group $=$ total cost $* \frac{\text { number of people with the disease in this age group }}{\text { Total population with the disease }}$

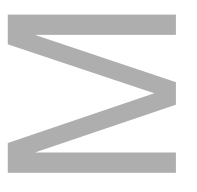

\section{Results}

\section{Projected prevalence of osteoarthritis to 2030}

In 2015, OA affected almost 2.2 million people (Figure 1). OA is by far the most common type of arthritis in Australia; in 2015, people with OA comprised $56.2 \%$ of the total arthritis population (estimated to be $3,904,306$ people). As shown in Figure 1, the number of people with OA is expected to increase nationally from almost 2.2 million in 2015 to almost 3.1 million Australians in the year 2030. People aged between 65-74 years represent the largest group with OA in absolute numbers, accounting for 631,345 people in 2015 (Table 1). The number of people with OA in this age group is expected to grow to almost 900,000 Australians by the year 2030. A sensitivity analysis incorporating alternative population projection scenarios produced similar findings, as shown in the supplementary material (Table S1). Under these alternative scenarios, the overall OA population in 2030 is estimated to range from 3.0 million people (low population growth scenario) to 3.1 million people (high population growth scenario).

This article is protected by copyright. All rights reserved 


\section{Projected prevalence of rheumatoid arthritis to $\mathbf{2 0 3 0}$}

RA is the second most prevalent form of arthritis in Australia and people with RA comprised $10.8 \%$ of the total arthritis population in 2015. Between 2015 and 2030, the number of people with RA in Australia is projected to increase from 422,309 people to 579,915 people (Table 2 ). In 2015 , the 65-74 age group had the greatest number of people with RA. With projected population growth, this age group is expected to continue to have the highest number of people with RA in the years 2020, 2025, and 2030. In the sensitivity analysis presented in Table S2 (Supplementary material), the overall RA population in 2030 is estimated to range from 574,021 (assuming a low population growth scenario) to 591,117 (assuming a high population growth scenario).

\section{Projected direct healthcare costs for osteoarthritis}

The direct healthcare costs for OA alone were estimated to be over $\$ 2.1$ billion in 2015 and by the year 2030, these are forecast to exceed $\$ 2.9$ billion (Table 3). In 2015, the highest healthcare costs for OA were related to the 65-74 age group (\$612 million) and this is projected to continue until the year 2030 (\$869 million). On average, this equates to $\$ 970$ in healthcare costs for every person with the condition.

\section{Projected direct healthcare costs for rheumatoid arthritis}

According to PBS expenditure data, benefits paid for bDMARDs indicated to treat RA in the 20142015 financial year totalled $\$ 273$ million (Supplementary material, Table S3). This represents a $104 \%$ increase from the costs reported in 2007 of $\$ 133.9$ million (25). As shown in Figure 2, the costs of bDMARDs alone are projected to increase to over $\$ 375$ million by the year 2030 , representing a $\$ 102$ million increase from 2015 costs.

Taking into account the costs for bDMARDs, healthcare costs for RA were estimated to be over $\$ 550$ million in 2015 (Table 4). This equates to an average of $\$ 1,303$ for each person with the condition. Healthcare costs for RA are projected to rise to over $\$ 755$ million by the year 2030 . The highest healthcare costs for RA were associated with the 65-74 age group in 2015 (\$147 million), and this pattern is projected to continue until the year 2030 (\$208 million).

\section{Discussion}

Our study provides national projections for the future prevalence of OA and RA in Australia, as well as projected direct healthcare costs, utilising contemporary data. It is clear that OA and RA are costly conditions that exert (and will increasingly exert) a substantial healthcare burden at the population level. Much of the predicted growth in direct healthcare costs relates to expected growth This article is protected by copyright. All rights reserved 
in the 65-74 and 75 and over age groups. While our projected costs are considerable, the full financial burden of treating OA and RA conditions is even greater given additional costs for nonmedical care, non-prescription medications and supplements, and out-of-pocket (non-reimbursed) costs for medical and surgical care. Studies of disease prevalence and cost of illness provide essential information to governments and other healthcare funders charged with healthcare planning and resource allocation. While the capacity of public healthcare systems to cope with projected growth remains unknown, our estimates provide tangible data that can assist in mapping future health service provision to expected need.

Our findings should be considered together with factors that may influence the future prevalence and healthcare costs associated with arthritis. The prevalence of obesity is increasing in Australia, and this may manifest in greater than anticipated growth in the prevalence of $O A$ and healthcare costs (related to more people living with the condition, greater difficulty in managing $O A$ in people who are obese, and the additional costs of co-morbidities related to obesity). The 2014-2015 National Health Survey showed that over $63 \%$ of Australian adults are overweight or obese $(36 \%$ are overweight and $28 \%$ are obese) (1). Using data from the national, population-based Australian Diabetes, Obesity and Lifestyle (AusDiab) Study, researchers predicted that the prevalence of obesity among adults will rise to $34 \%$ by the year 2025 (26). The future prevalence of OA and in particular, knee OA, may also be impacted by rising rates of sports-related joint injuries (27-29). A population-based study conducted in Victoria, Australia, showed a significant increase in sports injuries between the years 2004 and 2010, even after accounting for growth in sports participation (29). Lower limb sports-related injuries rose by $26 \%$ over the seven-year study period. Given the link between joint injury and subsequent development of knee OA (30), the researchers warned that the growth in sport-related lower injuries may signal a 'future epidemic of OA'.

While the advent of bDMARDs has changed the management of inflammatory arthritis, biologics are costly, as evidenced by our calculation of pharmaceutical costs for RA. Our projected costs are based on forecast growth in the RA population, with a relative increase in RA population size (and therefore bDMARD costs) of $12 \%$ from $2015-2020$, $11 \%$ from $2020-2025$, and $10 \%$ from 2025 2030. The development of biosimilar drugs (generic drugs that are designed to exert the same pharmacodynamics effects as other drugs) should lead to a decrease in treatment costs for RA (31). While generic medicines can typically offer an $80-90 \%$ reduction in costs from the originator drug, biosimilars are unlikely to decrease by this much due to the high cost of manufacturing. The introduction of biosimilars will result in an initial statutory reduction in cost of $16 \%$ in Australia (32), but this may reduce further over time through the PBS price disclosure program.

Our study has a number of strengths. We utilised the most up-to-date data on national arthritis prevalence and direct healthcare expenditure to forecast the future prevalence and costs of This article is protected by copyright. All rights reserved 
managing $O A$ and RA in Australia. In planning our analyses, we searched the peer-reviewed and grey literature and online resources, and identified and assembled a comprehensive set of national government data sources and published reports including national health survey data, national healthcare expenditure data, PBS expenditure data, and national population projections. All costs were updated to current pricing, to avoid underestimating the burden of arthritis when using expenditure data from earlier years. Additionally, we specifically considered government expenditure on bDMARDs indicated for RA using current PBS data. As pharmaceutical management is the backbone of RA treatment (nearly $80 \%$ of the total medical costs associated with RA in 2008-2009 were spent on prescription medications, equating to $\$ 275$ million (21)), this approach enabled us to more accurately quantify the true costs of healthcare for this disease.

We also acknowledge the main limitations associated with currently available data sources. The National Health Survey only included people living in private dwellings and did not include those living in nursing homes or other aged care facilities. This approach likely underestimates long-term health conditions that predominantly affect older people, such as OA. Additionally, the National Health Survey arthritis module captures doctor-diagnosed arthritis and this may underestimate true arthritis prevalence, by excluding people in the early stages of disease and/or those who have not yet been diagnosed. However, this approach is preferable to simply capturing 'self-reported arthritis', which could greatly overestimate the prevalence of arthritis. We acknowledge that there are issues with the specificity of self-reported arthritis questions (33) and as with any self-reported disease data, there is potential for misclassification bias. However, the case ascertainment methods used in the National Health Survey are consistent with those recommended by the Centers for Disease Control and Prevention in the United States for estimating arthritis prevalence in the general population (34). The methods are also consistent with those used for other largescale population health surveys around the world, as collection of radiographic and/or clinical data cannot be feasibly undertaken at the population level in order to estimate national arthritis prevalence. The AlHW health expenditure dataset does not capture the costs of follow-up appointments with medical specialists (including rheumatologists), as only the initial referral from a general practitioner is recorded. As RA requires close monitoring by a rheumatologist (with specialist review recommended 2-3 times per year (9)), actual out-of-hospital medical expenses are likely to be higher than presented in the AlHW report. The costs of allied health services (for example, visits to physiotherapists, occupational therapists and dieticians) are not covered within the AIHW's out-of-hospital expenses and would also add to the direct cost burden of arthritis. Our prevalence projections accounted for population growth and ageing over time but did not factor in potential increases in arthritis prevalence due to other factors, such as changes in obesity or sports injury rates. Finally, we acknowledge that our analyses do not account for any changes in treatment costs over time, for example as medications come 'off patent' or as new innovations in surgical management become available. Although challenging to undertake, future studies could

This article is protected by copyright. All rights reserved 
use a bottom-up costing approach involving population-based samples to capture the full costs of arthritis care (including the indirect costs of healthcare, such as time taken off work to attend appointments or for post-operative recovery).

In conclusion, almost 3.1 million Australians are expected to have OA by the year 2030, and over 579,000 people are predicted to have RA. Direct healthcare costs for OA and RA patient populations are forecast to exceed $\$ 2.9$ billion and $\$ 755$ million by 2030 , respectively. It is clear that $O A$ and RA are costly conditions that impose a substantial healthcare burden at the population level. These projections provide tangible data that can be utilised by health funders and policy makers to plan future health service provision.

\section{References}

1. Australian Bureau of Statistics. 4364.0.55.001 - National Health Survey: First results 2014-15. Available from: http://www.abs.gov.au/ausstats/abs@.nsf/mf/4364.0.55.001; accessed 4 April 2017.

2. Vos T, Barber RM, Bell B, Bertozzi-Villa A, Biryukov S, Bolliger I, et al. Global, regional, and national incidence, prevalence, and years lived with disability for 301 acute and chronic diseases and injuries in 188 countries, 1990-2013: A systematic analysis for the Global Burden of Disease Study 2013. Lancet 2015;386:743-800.

3. Cross M, Smith E, Hoy D, Nolte S, Ackerman I, Fransen M, et al. The global burden of hip and knee osteoarthritis: Estimates from the Global Burden of Disease 2010 study. Ann Rheum Dis 2014;73:1323-1330.

4. Cross M, Smith E, Hoy D, Carmona L, Wolfe F, Vos T, et al. The global burden of rheumatoid arthritis: Estimates from the Global Burden of Disease 2010 study. Ann Rheum Dis 2014;73:1316-1322.

5. Royal Australian College of General Practitioners. Guideline for the non-surgical management of hip and knee osteoarthritis. Melbourne: Royal Australian College of General Practitioners; 2009.

6. Zhang W, Nuki G, Moskowitz RW, Abramson S, Altman RD, Arden NK, et al. OARSI recommendations for the management of hip and knee osteoarthritis: Part III: Changes in evidence following systematic cumulative update of research published through January 2009. Osteoarthritis Cartilage 2010;18:476-499.

7. Fernandes L, Hagen KB, Bijlsma JWJ, Andreassen O, Christensen P, Conaghan PG, et al. EULAR recommendations for the non-pharmacological core management of hip and knee osteoarthritis. Ann Rheum Dis 2013;72:1125-1135.

8. National Institute for Health and Care Excellence. Osteoarthritis: Care and management. Available from: https://www.nice.org.uk/guidance/cg177/resources/osteoarthritis-care-and-management35109757272517; accessed 4 April 2017.

9. Royal Australian College of General Practitioners. Clinical guideline for the diagnosis and management of early rheumatoid arthritis. Melbourne: Royal Australian College of General Practitioners; 2009.

This article is protected by copyright. All rights reserved 
10. National Institute for Health and Care Excellence. Rheumatoid arthritis in adults: management. Available from: https://www.nice.org.uk/guidance/cg79/resources/rheumatoid-arthritis-in-adultsmanagement-975636823525; accessed 4 April 2017.

11. Smolen JS, Landewe R, Burmester G, Dougados M, Emery P, Gaujoux-Viala C, et al. EULAR recommendations for the management of rheumatoid arthritis with synthetic and biological diseasemodifying antirheumatic drugs: 2013 update. Ann Rheum Dis 2014;73:492-509.

12. Singh JA, Saag KG, Bridges SL, Jr., Akl EA, Bannuru RR, Sullivan MC, et al. 2015 American College of Rheumatology guideline for the treatment of rheumatoid arthritis. Arthritis Rheumatol 2015;68:1-26.

13. Access Economics. Painful realities: The economic impact of arthritis in Australia in 2007. Sydney: Arthritis Australia; 2007.

14. Arthritis and Osteoporosis Victoria. A problem worth solving: The rising cost of musculoskeletal conditions in Australia. Melbourne: Arthritis and Osteoporosis Victoria; 2013.

15. Australian Orthopaedic Association National Joint Replacement Registry. Annual report. Adelaide; 2001.

16. Australian Orthopaedic Association National Joint Replacement Registry. Annual report - Hip, knee and shoulder arthroplasty. Adelaide: Australian Orthopaedic Association; 2016.

17. Meier FM, Frerix M, Hermann W, Muller-Ladner U. Current immunotherapy in rheumatoid arthritis. Immunotherapy 2013;5:955-974.

18. Australian Bureau of Statistics. 4363.0.55.001 - Australian Health Survey: Users' Guide, 2011-13. Available from: http://www.abs.gov.au/ausstats/abs@.nst/PrimaryMainFeatures/4363.0.55.001?OpenDocument; accessed 28 July 2017.

19. Australian Bureau of Statistics. Dataset: Population Projections, Australia, 2012-2101. Available from: http://stat.data.abs.gov.au/Index.aspx?DataSetCode=POP_PROJ_2011; accessed 28 July 2017.

20. Australian Bureau of Statistics. 3222.0 Population Projections, Australia, 2012 (base) to 2101. Available from: http://www.abs.gov.au/Ausstats/abs@.nsf/mf/3222.0; accessed 28 July 2017.

21. Australian Institute of Health and Welfare. Health-care expenditure on arthritis and other musculoskeletal conditions 2008-09. Canberra: Australian Institute of Health and Welfare; 2014.

22. Britt HC, Miller GC. The Bettering the Evaluation and Care of Health (BEACH) program: Where to from here? Med J Aust 2013;198:125-126.

23. Department of Human Services. Pharmaceutical Benefits Schedule Item Reports. Available from: http://medicarestatistics.humanservices.gov.au/statistics/pbs_item.jsp; accessed 4 April 2017.

24. Australian Institute of Health and Welfare. Table E2: Total health price index and industry-wide indexes. Available from: http://www.aihw.gov.au/WorkArea/DownloadAsset.aspx?id=6442473791; accessed 4 Aprit 2017.

25. Australian Institute of Health and Welfare. The use of disease-modifying anti-rheumatic drugs for the management of rheumatoid arthritis. Canberra: Australian Institute of Health and Welfare; 2011.

26. Walls HL, Magliano DJ, Stevenson CE, Backholer K, Mannan HR, Shaw JE, et al. Projected progression of the prevalence of obesity in Australia. Obesity 2012;20:872-878.

27. Roos EM. Joint injury causes knee osteoarthritis in young adults. Curr Opin Rheumatol 2005;17:195200.

This article is protected by copyright. All rights reserved 
28. Lohmander LS, Englund PM, Dahl LL, Roos EM. The long-term consequence of anterior cruciate ligament and meniscus injuries: Osteoarthritis. Am J Sports Med 2007;35:1756-1769.

29. Finch CF, Kemp JL, Clapperton AJ. The incidence and burden of hospital-treated sports-related injury in people aged 15+ years in Victoria, Australia, 2004-2010: A future epidemic of osteoarthritis? Osteoarthritis Cartilage 2015;23:1138-1143.

30. Ackerman IN, Kemp JL, Crossley KM, Culvenor AG, Hinman RS. Hip and knee osteoarthritis affects younger people, too. J Orthop Sports Phys Ther 2017;47:67-79.

31. Reinaud F, Ando G. Price differences triggered by the availability of biosimilars in developed countries. Value Health 2013;16:A329.

32. Department of Health. Pharmaceutical Benefits Scheme: $12.5 \%$ and $16 \%$ Price Reductions - 1 April 2016. Available from: http://www.pbs.gov.au/info/industry/pricing/pbs-items/price-reductions; accessed 4 April 2017.

33. Sacks JJ, Harrold LR, Helmick CG, Gurwitz JH, Emani S, Yood RA. Validation of a surveillance case definition for arthritis. J Rheumatol 2005;32:340-347.

34. Centers for Disease Control and Prevention. Arthritis case definitions. Available from: https://www.cdc.gov/arthritis/data_statistics/case_definition.htm; accessed 28 July 2017.

Table 1. Projected national prevalence of osteoarthritis to 2030, by age group

\begin{tabular}{|r|c|r|r|r|r|}
\hline Age group & OA prevalence & $\mathbf{2 0 1 5}^{\star}$ & $\mathbf{2 0 2 0}^{\star}$ & $\mathbf{2 0 2 5}^{\star}$ & $\mathbf{2 0 3 0}^{*}$ \\
\hline $\mathbf{1 5} \mathbf{- 2 4}$ & $0.4 \%$ & 12,528 & 12,904 & 13,761 & 14,961 \\
\hline $\mathbf{2 5} \mathbf{- 3 4}$ & $1.1 \%$ & 39,099 & 41,781 & 42,918 & 43,822 \\
\hline $\mathbf{3 5} \mathbf{- 4 4}$ & $3.1 \%$ & 101,356 & 110,480 & 124,547 & 131,818 \\
\hline $\mathbf{4 5} \mathbf{- 5 4}$ & $9.6 \%$ & 301,608 & 317,599 & 331,099 & 358,809 \\
\hline $\mathbf{5 5}-\mathbf{6 4}$ & $22.1 \%$ & 606,672 & 663,302 & 692,738 & 729,651 \\
\hline $\mathbf{6 5}-\mathbf{7 4}$ & $31.4 \%$ & 631,345 & 736,561 & 815,613 & 896,039 \\
\hline $\mathbf{7 5}$ years & $32.3 \%$ & 502,792 & 593,552 & 737,431 & 882,561 \\
\hline TOTAL & & $\mathbf{2 , 1 9 5 , 4 0 0}$ & $\mathbf{2 , 4 7 6 , 1 7 9}$ & $\mathbf{2 , 7 5 8 , 1 0 7}$ & $\mathbf{3 , 0 5 7 , 6 6 1}$ \\
\hline
\end{tabular}

*Data presented as the number of people with osteoarthritis

While children aged 0-14 years were included in the National Health Survey, data for this age group are not presented given that the prevalence of OA for this group was $0.0 \%$

Table 2. Projected national prevalence of rheumatoid arthritis to 2030 , by age group

\begin{tabular}{|r|c|c|c|c|c|}
\hline Age group & RA prevalence & $\mathbf{2 0 1 5}^{\boldsymbol{}}$ & $\mathbf{2 0 2 0}^{\star}$ & $\mathbf{2 0 2 5}^{\boldsymbol{*}}$ & $\mathbf{2 0 3 0}^{\boldsymbol{*}}$ \\
\hline $\mathbf{1 5} \mathbf{- 2 4}$ & $0.6 \%$ & 18,792 & 19,355 & 20,641 & 22,442 \\
\hline
\end{tabular}

This article is protected by copyright. All rights reserved 


\begin{tabular}{|r|r|r|r|r|r|}
\hline $\mathbf{2 5}-\mathbf{3 4}$ & $0.3 \%$ & 10,664 & 11,395 & 11,705 & 11,952 \\
\hline $\mathbf{3 5}-\mathbf{4 4}$ & $1.2 \%$ & 39,235 & 42,767 & 48,212 & 51,027 \\
\hline $\mathbf{4 5}-\mathbf{5 4}$ & $1.9 \%$ & 59,694 & 62,859 & 65,530 & 71,015 \\
\hline $\mathbf{5 5}-\mathbf{6 4}$ & $3.6 \%$ & 98,825 & 108,050 & 112,845 & 118,858 \\
\hline $\mathbf{6 5}-\mathbf{7 4}$ & $5.6 \%$ & 112,597 & 131,362 & 145,460 & 159,804 \\
\hline $\mathbf{7 5}$ years & $5.3 \%$ & 82,502 & 97,394 & 121,003 & 144,817 \\
\hline TOTAL & & $\mathbf{4 2 2 , 3 0 9}$ & $\mathbf{4 7 3 , 1 8 2}$ & $\mathbf{5 2 5 , 3 9 6}$ & $\mathbf{5 7 9 , 9 1 5}$ \\
\hline
\end{tabular}

*Data presented as the number of people with rheumatoid arthritis

While children aged 0-14 years were included in the National Health Survey, data for this age group are not presented given that the prevalence of RA for this group was $0.0 \%$

Table 3. Projected healthcare costs for osteoarthritis to 2030, by age group

\begin{tabular}{|r|r|r|r|r|r|r|}
\hline Age group & $\mathbf{2 0 1 5}$ & $\mathbf{2 0 2 0}$ & $\mathbf{2 0 2 5}$ & $\mathbf{2 0 3 0}$ & $\begin{array}{c}\text { Difference } \\
\mathbf{( 2 0 1 5 - 2 0 3 0 )}\end{array}$ & $\begin{array}{c}\text { \% change } \\
\mathbf{( 2 0 1 5 - 2 0 3 0 )}\end{array}$ \\
\hline $\mathbf{1 5 - 2 4}$ & 12.16 & 12.52 & 13.35 & 14.52 & 2 & $19.4 \%$ \\
\hline $\mathbf{2 5} \mathbf{- 3 4}$ & 37.94 & 40.54 & 41.65 & 42.52 & 5 & $12.1 \%$ \\
\hline $\mathbf{3 5}-\mathbf{4 4}$ & 98.35 & 107.21 & 120.86 & 127.91 & 30 & $30.1 \%$ \\
\hline $\mathbf{4 5}-\mathbf{5 4}$ & 292.67 & 308.19 & 321.29 & 348.18 & 56 & $19.0 \%$ \\
\hline $\mathbf{5 5}-\mathbf{6 4}$ & 588.70 & 643.65 & 672.22 & 708.04 & 119 & $20.3 \%$ \\
\hline $\mathbf{6 5}-\mathbf{7 4}$ & 612.64 & $\mathbf{7 1 4 . 7 4}$ & 791.45 & 869.50 & 257 & $41.9 \%$ \\
\hline $\mathbf{7 5}$ years & 487.90 & 575.97 & 715.59 & 856.42 & 369 & $75.5 \%$ \\
\hline TOTAL & $\mathbf{2 , 1 3 0 . 3 6}$ & $\mathbf{2 , 4 0 2 . 8 2}$ & $\mathbf{2 , 6 7 6 . 4 1}$ & $\mathbf{2 , 9 6 7 . 0 9}$ & $\mathbf{8 3 7}$ & $\mathbf{3 9 . 3} \%$ \\
\hline
\end{tabular}

All costs are reported in \$AUD 000,000 (\$AUD 1.00 is equivalent to approximately \$US 0.76)

While children aged 0-14 years were included in the National Health Survey, data for this age group are not presented given that the prevalence of OA for this group was $0.0 \%$

Table 4. Projected healthcare costs for rheumatoid arthritis to 2030 , by age group

\begin{tabular}{|r|c|c|c|c|c|c|}
\hline Age group & 2015 & 2020 & 2025 & 2030 & $\begin{array}{c}\text { Difference } \\
(\mathbf{2 0 1 5 - 2 0 3 0 )}\end{array}$ & $\begin{array}{c}\text { \% change } \\
\mathbf{( 2 0 1 5 - 2 0 3 0 )}\end{array}$ \\
\hline $\mathbf{1 5 - 2 4}$ & 24.49 & 25.22 & 26.90 & 29.25 & 5 & $19.4 \%$ \\
\hline
\end{tabular}

This article is protected by copyright. All rights reserved 


\begin{tabular}{|r|r|r|r|r|r|r|}
\hline $\mathbf{2 5}-\mathbf{3 4}$ & 13.90 & 14.85 & 15.25 & 15.58 & 2 & $12.1 \%$ \\
\hline $\mathbf{3 5}-\mathbf{4 4}$ & 51.13 & 55.74 & 62.83 & 66.50 & 15 & $30.1 \%$ \\
\hline $\mathbf{4 5} \mathbf{- 5 4}$ & $\mathbf{7 7 . 8 0}$ & 81.92 & 85.40 & 92.55 & 15 & $19.0 \%$ \\
\hline $\mathbf{5 5}-\mathbf{6 4}$ & 128.79 & 140.81 & 147.06 & 154.90 & 26 & $20.3 \%$ \\
\hline $\mathbf{6 5}-\mathbf{7 4}$ & 146.74 & 171.20 & 189.57 & 208.26 & 62 & $41.9 \%$ \\
\hline $\mathbf{7 5}$ years & 107.52 & 126.93 & 157.70 & 188.73 & 81 & $\mathbf{7 5 . 5 \%}$ \\
\hline TOTAL & $\mathbf{5 5 0 . 3 7}$ & $\mathbf{6 1 6 . 6 7}$ & $\mathbf{6 8 4 . 7 1}$ & $\mathbf{7 5 5 . 7 7}$ & $\mathbf{2 0 5}$ & $\mathbf{3 7 . 3} \%$ \\
\hline
\end{tabular}

All costs are reported in \$AUD 000,000 (\$AUD 1.00 is equivalent to approximately \$US 0.76)

While children aged 0-14 years were included in the National Health Survey, data for this age group are not presented given that the prevalence of RA for this group was $0.0 \%$

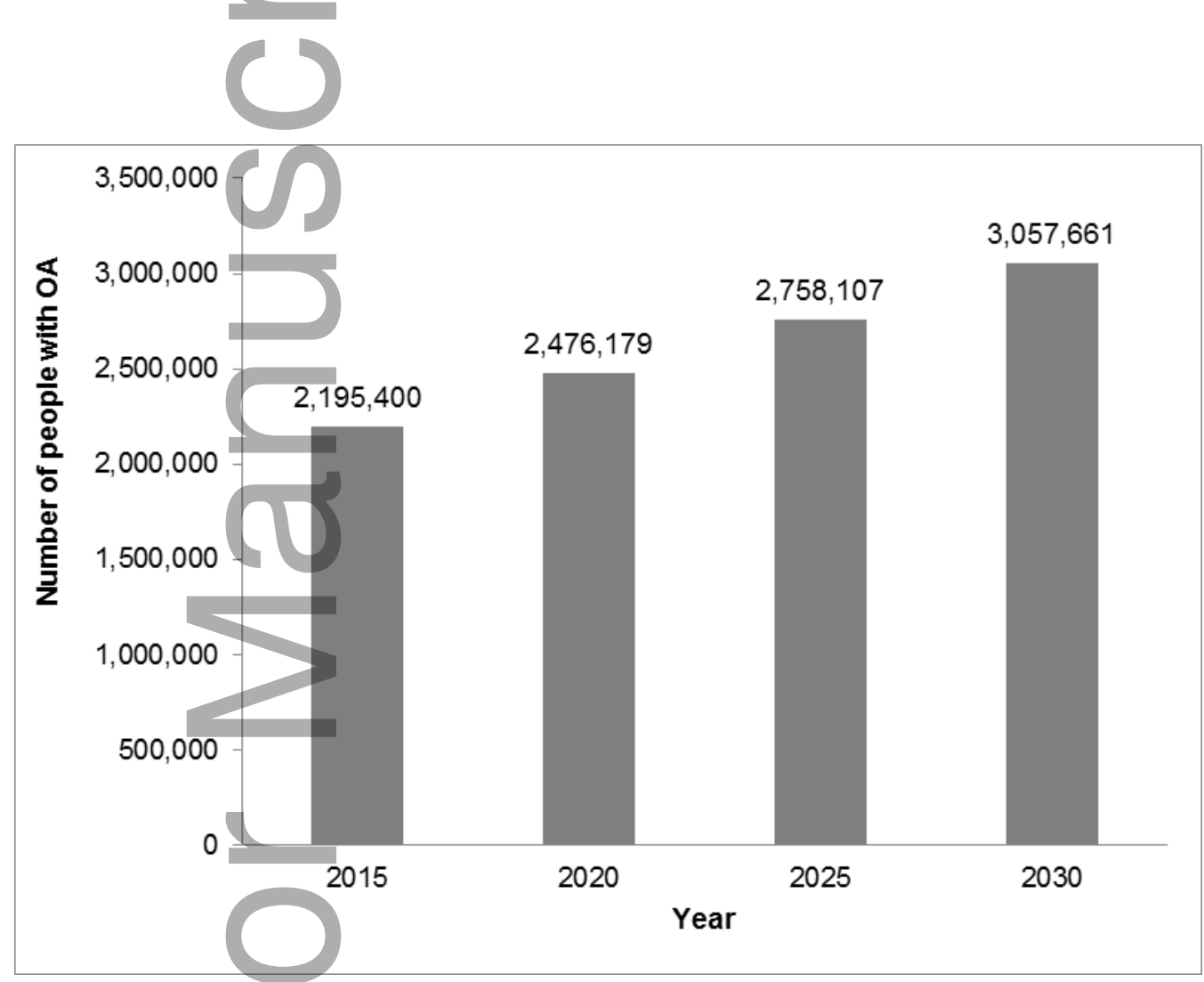

Figure 1. Projected national prevalence of osteoarthritis in Australia, 2015-2030

This article is protected by copyright. All rights reserved 


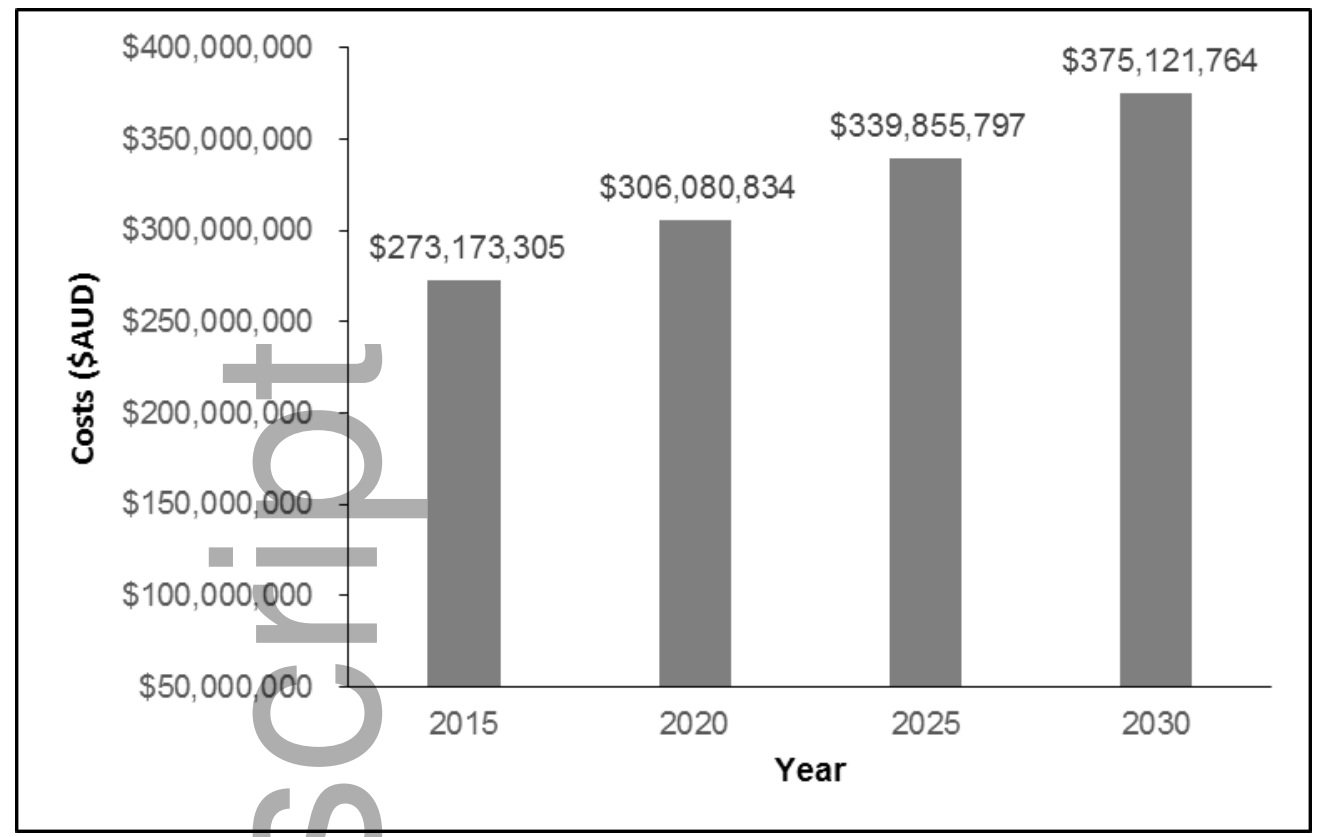

Figure 2. Projected costs of biologic DMARDs for rheumatoid arthritis to 2030

All costs are reported in \$AUD (\$AUD 1.00 is equivalent to approximately $\$ \cup S$ 0.76)

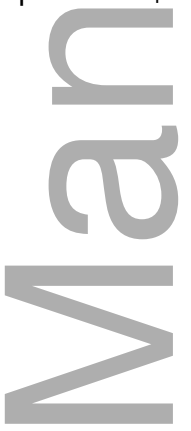

\section{Figure legends}

Figure 1. Projected national prevalence of osteoarthritis in Australia, 2015-2030

Figure 2. Projected costs of biologic DMARDs for rheumatoid arthritis to 2030

All costs are reported in \$AUD (\$AUD 1.00 is equivalent to approximately \$US 0.76)

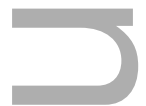

\section{Acknowledgments}

We gratefully acknowledge the contribution of Dr Megan Bohensky (formerly The University of Melbourne) to research design, project management, data analysis and preparation of earlier reports, and the support provided by Franca Marine (Arthritis Australia) in developing this research. 


\section{Conflict of interest}

The authors declare there are no conflicts of interest with respect to this manuscript.

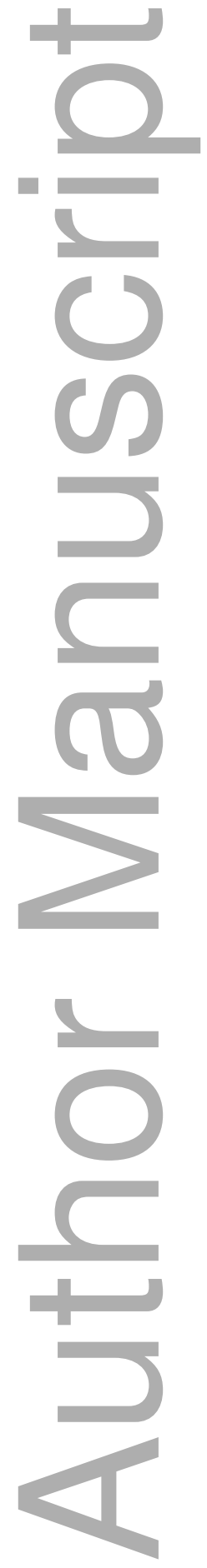

This article is protected by copyright. All rights reserved 


\section{University Library}

\section{- M M N E R VA A gateway to Melbourne's research publications}

Minerva Access is the Institutional Repository of The University of Melbourne

Author/s:

Ackerman, IN;Pratt, C;Gorelik, A;Liew, D

Title:

Projected Burden of Osteoarthritis and Rheumatoid Arthritis in Australia: A Population-Level Analysis

Date:

2017-09-12

Citation:

Ackerman, I. N., Pratt, C., Gorelik, A. \& Liew, D. (2017). Projected Burden of Osteoarthritis and Rheumatoid Arthritis in Australia: A Population-Level Analysis. ARTHRITIS CARE \& RESEARCH, 70 (6), pp.877-883. https://doi.org/10.1002/acr.23414.

Persistent Link:

http://hdl.handle.net/11343/293445 